\title{
ZEB1-induced LINC01559 expedites cell proliferation, migration and EMT process in gastric cancer through recruiting IGF2BP2 to stabilize ZEB1 expression
}

\author{
Huojian Shen ${ }^{1}$, Hongyi Zhu', Yuanwen Chen', Zhiyong Shen', Weiqing Qiu', Changlin Qian ${ }^{1}$ and Jie Zhang ${ }^{1}$
}

\begin{abstract}
Gastric cancer (GC) is a common type of tumor that is characterized with high metastatic rate. In recent years, increasing studies have indicated that IncRNAs are involved in the regulation on cancer cell proliferation and migration. However, the functional role of long intergenic non-protein coding RNA 1559 (LINC01559) in GC is still unclear. In this study, we applied quantitative real-time polymerase chain reaction (RT-qPCR) and examined that LINC01559 expression was significantly enhanced in GC cells. Functional assays such as EdU, colony formation, JC-1 and transwell assays displayed that silencing LINC01559 inhibited cell proliferation and migration while promoted cell apoptosis in GC. Besides, western blot analysis and immunofluorescence assays examined the expression of factors related to epithelialmesenchymal transition (EMT) and indicated that EMT process was blocked by LINC01559 knockdown in GC cells. Besides, LINC01559 silencing inhibited tumor growth in vivo. In addition, Chromatin immunoprecipitation (ChIP) assays demonstrated that zinc finger E-box binding homeobox 1 (ZEB1) served as a transcription factor to combine with LINC01559 promoter and activated the expression of LINC01559 in GC cells. In return, LINC01559 recruited insulin like growth factor 2 mRNA binding protein 2 (IGF2BP2) to stabilize ZEB1 mRNA to up-regulate ZEB1 in GC cells. In short, the findings in this research might provide a novel target for GC treatment.
\end{abstract}

\section{Introduction}

Nowadays, gastric cancer (GC) is one of the most common cancers worldwide ${ }^{1}$. In East Asia, especially in Korea, Mongolia, Japan, and China, GC-associated morbidity and mortality have been shown to be relatively higher than in most western countries ${ }^{2,3}$. Despite advances in diagnostic techniques and improvements in chemotherapy and radiotherapy, patients with advanced or metastatic GC still present a poor prognosis ${ }^{4}$. One of the main causes of cancer-related mortality is tumor

\footnotetext{
Correspondence: Jie Zhang (Zhangjie153000@163.com)

${ }^{1}$ Renji Hospital Affiliated to Medical College of Shanghai Jiaotong University, Shanghai 200025, China

These authors contributed equally: Huojian Shen, Hongyi Zhu

Edited by G. Calin
}

metastasis. Therefore, early diagnosis of GC is of significant importance for prognosis 5 .

Long non-coding RNAs (lncRNAs) are a type of discovered RNAs with the length over 200 nucleotides and without the ability of coding proteins ${ }^{6,7}$. LncRNAs are involved in a wide range of biological processes in various cancers, such as cell proliferation, migration and apoptosis $^{8}$. Abnormally expressed lncRNAs can serves as oncogenes or tumor suppressors to affect the progression and metastasis of cancers ${ }^{9,10}$. Mechanistically, lncRNAs exert their functions majorly through the interaction with RNAs or proteins in the regulation of gene expression ${ }^{11}$. In recent years, a large number of studies have shown that lncRNAs play vital roles in the progression of GC, such as MEG3 ${ }^{12}$, HOXA11-AS ${ }^{13}$ and LINC00337 ${ }^{14}$. However, there are still

\section{(c) The Author(s) 2021}

(c) (i) Open Access This article is licensed under a Creative Commons Attribution 4.0 International License, which permits use, sharing, adaptation, distribution and reproduction c. in any medium or format, as long as you give appropriate credit to the original author(s) and the source, provide a link to the Creative Commons license, and indicate if changes were made. The images or other third party material in this article are included in the article's Creative Commons license, unless indicated otherwise in a credit line to the material. If material is not included in the article's Creative Commons license and your intended use is not permitted by statutory regulation or exceeds the permitted use, you will need to obtain permission directly from the copyright holder. To view a copy of this license, visit http://creativecommons.org/licenses/by/4.0/. 
some unknown lncRNAs whose mechanisms need to be further explored in GC.

Zinc finger E-box binding homeobox 1 (ZEB1) is a transcription factor registered by some studies. In prostate cancer cells, ZEB1 is a key promoter of tumor progres$\operatorname{sion}^{15}$. In breast cancer cells, ZEB1 induces transcriptional regulation of genes involved in the cancer progression ${ }^{16}$. Thus, it is of great interest and importance to identify the association between IncRNAs and ZEB1 in the progression of GC.

In addition to target and sequester specific microRNAs (miRNAs), lncRNAs can function in the progression of cancers through recruiting RNA-binding proteins $(\mathrm{RBPs})^{17}$. Abundant studies have demonstrated the participation of RBPs in the maintenance of mRNA stability by lncRNAs in cancer cells. For instance, CERS6-AS1 promotes the progression and metastasis of breast cancer by recruiting with IGF2BP3 to increase the stability of CERS6 mRNA $^{18}$. Besides, LIN28B-AS1 interacts with by IGF2BP1 to activate LIN28B expression in lung adenocarcinoma cells $^{19}$. In our study, we also investigated whether LINC01559 could recruit certain protein partner to exert its function in GC cells.

Epithelial-to-mesenchymal transition (EMT) is a cellular process related to metastasis ${ }^{20}$. EMT is essential for embryonic development and has been associated with a variety of diseases. EMT-related transcription factors (Snail, Twist, and ZEB1) can induce EMT process in vitro and in vivo ${ }^{21}$. However, whether and how EMT is modulated by LINC01559 in GC cells remain to be further specified.

In conclusion, our study expounded that LINC01559 expression was up-regulated in GC cells. Knockdown of LINC01559 inhibited cell proliferation, migration and EMT process in GC. Moreover, ZEB1 could activate the expression of LINC01559, and LINC01559 recruited insulin like growth factor 2 mRNA binding protein 2 (IGF2BP2) to stabilize ZEB1 mRNA in GC cells. In a word, this study might be helpful to identify effective treatments for GC.

\section{Materials and methods \\ Cell culture}

Human GC cell lines (SNU-1 and AGS) were purchased from American Type Culture Collection (ATCC) cell bank (Manassas, VA); another two kinds of human GC cell lines (MKN45 and HGC-27) were gotten from the Institute of Biochemistry and Cell Biology, Chinese Academy of Sciences (Shanghai, China); the normal human gastric epithelial cell line (GES-1) was obtained from Procell Life Science \& Technology Co., Ltd. (Wuhan, China). SNU-1, MKN45, HGC-27 and GES-1 cells were cultivated in RPMI-1640 medium (Invitrogen, Carlsbad, CA, USA). AGS cells was cultivated in F-12K medium (Invitrogen, Carlsbad, CA, USA). The culture media were all supplemented with $10 \% \mathrm{FBS}$ and $1 \% \mathrm{P} / \mathrm{S}$. All cells were grown in a humidified atmosphere of $5 \%$ $\mathrm{CO}_{2}$ at $37^{\circ} \mathrm{C}$.

\section{Cell transfection}

Specific shRNAs targeting LINC01559 (sh/ LINC01559\#1/\#2) or IGF2BP2 (sh/IGF2BP2\#1/\#2) and control group (sh/Ctrl) were synthesized by GenePharma (Shanghai, China). The pcDNA3.1 vectors from Invitrogen were utilized to construct pcDNA3.1/ZEB1, pcDNA3.1/ MAFK, pcDNA3.1/JUND, pcDNA3.1/JUN, pcDNA3.1/ ESR1 and pcDNA3.1/RFX5, with the empty vector as the negative control. Lipofectamine 3000 (Invitrogen) was used to execute transfection experiments for $48 \mathrm{~h}$.

\section{Quantitative real-time polymerase chain reaction (RT- qPCR)}

Total RNA was extracted from GC cells using the TRIzol reagent according to manufacturer's requirements (Thermo Fisher Scientific), and then cDNA was obtained after reverse transcription. RT-qPCR was performed with SYBR Green Master Mixture (Takara, Dalian, China). GAPDH was used as the control. Relative gene expression levels were calculated using the $2^{-\Delta \Delta \mathrm{Ct}}$ method. The experiment was repeated for three times.

\section{5-ethynyl-2'-deoxyuridine (EdU) assay}

After transfection, GC cells were seeded onto 96-well plates. The EdU (5-ethynyl-20-deoxyuridine) Apollo567 Kit (RIBOBIO, Shanghai, China) was used to quantify the proliferation ability of indicated GC cells. EdU and DAPI dyes were exploited to treat GC cells. A fluorescent microscope was used for cell visualization (Olympus, Tokyo, Japan). The experiment was repeated for three times.

\section{Colony formation assay}

GC cells were seeded into 6-well plate at the density of 500 cells per well. After 2 weeks, colonies were fixed with $4 \%$ paraformaldehyde and stained with $0.1 \%$ crystal violet (DINGGUO) and then counted manually. The experiment was repeated for three times.

\section{Flow cytometry analysis}

The apoptosis rate of SNU-1 and AGS cells was analyzed with propidium iodide (PI)/Annexin V Cell Apoptosis Kit (Invitrogen, V13245) in reference to manufacturer's suggestions. In short, after $48 \mathrm{~h}$ transfection, SNU-1 and AGS cells were stained by FITC-Annexin V and PI. Finally, cell apoptosis rate was examined by flow cytometry (FACSCantoII, 338960; BD Biosciences, San Jose, CA, USA). The experiment was repeated for three times. 


\section{JC-1 assay}

Cells were incubated with $500 \mu \mathrm{l} \mathrm{JC-1} \mathrm{staining} \mathrm{working}$ solution for $20 \mathrm{~min}$ at $37^{\circ} \mathrm{C}$ (Beyotime, China). The fluorescence labeled cells were washed using phosphate buffered saline (PBS) and analyzed by an EnSpire Reader. The data and images were processed by GraphPad Prism 6.0 statistical software. The experiment was repeated for three times.

\section{Transwell assay}

GC cells $\left(1 \times 10^{5}\right)$ in serum-free medium were seeded on the upper transwell chamber (BD Biosciences). The complete culture medium (with 10\% FBS) was added to the lower chamber. After $24 \mathrm{~h}$, the migrated cells on the lower surface were fixed and stained. Five repeated views in each condition were used to calculate the number of migrated cells. The experiment was repeated for three times.

\section{Western blot}

Total cellular lysates were resolved by RIPA lysis buffer and then proteins were separated via SDS-PAGE (10\%) gels and transferred to a PVDF membrane. The latter was incubated with blocking buffer and followed by further processing with primary antibodies against GAPDH (1/ 10000, ab181602), E-cadherin (1:2000-1:8000, 60335-1Ig), N-cadherin $(1 \mu \mathrm{g} / \mathrm{ml}$, ab18203), Slug (1:1000, 9585S), Twist (1/5000, ab187008), ZEB1 (1:500-1:2000, 21544-1AP), IGF2BP2 (1:1000, 14672S), MAFK (1/4000-1/8000, ab50322), JUND (1:1000, 5000S), JUN (1:1000-1:8000, 66313-1-Ig), ESR1 (1:500-1:2000, 21244-1-AP) and RFX5 (1/500-1/5000, ab9255) as well as secondary antibodies. ECL method was applied to detect the immuno-reactive bands. The experiment was repeated for three times.

\section{Immunofluorescence analysis}

SNU-1 and AGS cells were fixed with $4 \%$ paraformaldehyde, followed by the incubation with primary antibodies against E-cadherin (1:2000-1:8000, 60335-1-Ig) and N-cadherin $\left(1 \mu \mathrm{g} / \mathrm{ml}\right.$, ab18203) overnight at $4{ }^{\circ} \mathrm{C}$. Then cells were hatched with $\mathrm{Cy} 3$-conjugated secondary antibody for $30 \mathrm{~min}$ at room temperature. The nuclei were re-stained by DAPI for $3 \mathrm{~min}$. The images were gotten via a fluorescence microscopy (Nikon Corporation) and qualified by Image-Pro Plus software. The experiment was repeated for three times.

\section{Tumor xenograft assay}

Four-week-old female athymic nude mice were purchased from Renji Hospital Affiliated to Medical College of Shanghai Jiaotong University. This study was approved by the Ethic Committee of Renji Hospital Affiliated to Medical College of Shanghai Jiaotong University. $1 \times 10^{7}$ cells transfected with sh/Ctrl or sh/LINC01559\#1 were injected subcutaneously into the randomly divided two groups of nude mice. The tumor volume was tested every four days and tumor growth lasted for 4 weeks. After that, the mice were mercy killing and their tumors were taken out. The tumor weight was calculated and the tumors were collected for following analysis.

\section{Immunohistochemical (IHC) assay}

The collected tumor tissues from xenograft assay were dehydrated after fixing in $4 \%$ paraformaldehyde, and then embedded in paraffin and cut into sections of $4 \mu \mathrm{m}$ thick. Then the sections were utilized for IHC assay by use of specific antibody to Ki-67, PCNA, E-cadherin or Ncadherin (Abcam, Cambridge, MA). The experiment was repeated for three times.

\section{Chromatin immunoprecipitation (ChIP) assay}

ChIP analysis was carried out using the Millipore ChIP Assay Kit (Millipore, MA, USA) based on the manufacturer's instructions. SNU-1 and AGS cells were cross-linked with formaldehyde and sonicated into 200-1000 bp. DNA samples were precipitated with antiIgG or anti-ZEB1 antibody upon $30 \mu \mathrm{l}$ of magnetic beads for $6 \mathrm{~h}$. Then, the precipitated chromatin DNA was collected, extracted and purified for RT-qPCR analysis. The experiment was repeated for three times.

\section{Luciferase reporter assay}

The sequence of wild-type or indicated site-mutated LINC01559 promoter (WT, Site 1-MUT, Site 2-MUT, Site 3-MUT, Site 4-MUT, Site 5-MUT, and MUT) were synthesized and cloned into pGL3 vector to construct corresponding reporter plasmids. Then, indicated recombinant reporter was co-transfected with pcDNA3.1/ ZEB1 or pcDNA3.1 into SNU-1 and AGS cells. After $48 \mathrm{~h}$, the luciferase activity of each group was detected using Dual-Luciferase Reporter Assay System (Promega). The experiment was repeated for three times.

\section{Fluorescence in situ Hybridization (FISH) assay}

Fluorescently-labeled LINC01559 probe was constructed by RiboBio. The FISH Kit (RiboBio) was used for the detection of LINC01559 signals. DAPI was utilized to re-stain the nuclei of SNU-1 and AGS cells. Images were acquired through a confocal microscope (Olympus, Tokyo, Japan). The experiment was repeated for three times.

\section{Subcellular fractionation}

Nuclear or cytoplasmic RNA in SNU-1 and AGS cells was separated and purified with the application of a Cytoplasmic and Nuclear RNA Purification Kit (Norgen Biotek, Thorold, Canada). The percentage of LINC01559 in nuclear or cytoplasm fractions was examined with RT-qPCR. GAPDH and U6 were the control for 
cytoplasmic or nuclear RNA, respectively. The experiment was repeated for three times.

\section{Actinomycin D experiment}

SNU-1 and AGS cells were seeded in 24-well plates $(5 \times$ $10^{4} /$ well). After transfection, cells were exposed to Actinomycin D $(2 \mu \mathrm{g} / \mathrm{ml}$, Abcam, ab141058) for $0 \mathrm{~h}, 4 \mathrm{~h}$ and $8 \mathrm{~h}$. Then the relative mRNA level of ZEB1 was examined by RT-qPCR and normalized to the values measured in the $0 \mathrm{~h}$ group (mock treatment). The experiment was repeated for three times.

\section{RNA pull-down assay}

Biotin-labeled full-length and antisense LINC01559 or ZEB1 sequences were obtained by the use of Transcript Aid T7 High Yield Transcription Kit (Thermo Scientific). Then the MEGAclearTM Kit (Thermo Scientific) was applied to recycle the sequences in line with manufacturer's advice. The sequences were incubated with cell lysates for $4 \mathrm{~h}$ at $4{ }^{\circ} \mathrm{C}$, and then the biotin-labeled RNAs and their binding protein partner were pulled down by streptavidin magnetic beads (Thermo, USA) at $4{ }^{\circ} \mathrm{C}$ overnight. The proteins were separated by electrophoresis and visualized using the Coomassie Blue Staining Kit (Beyotime, China). The different bands between sense and antisense LINC01559 were identified using mass spectrometry and determined through western blot analysis. The experiment was repeated for three times.

\section{RNA immunoprecipitation (RIP) assays}

RIP assay was conducted to study whether LINC01559 or ZEB1 interacted with IGF2BP2 by using a Magna RIP RNA-binding protein immunoprecipitation kit (Millipore, Billerica, MA, USA). Cells were lysed with RIP buffer, and then the cell extract was mixed with agarose beads carrying anti-IGF2BP2 or anti-lgG antibody. The enrichment of co-precipitated RNAs was detected using RT-qPCR. The experiment was repeated for three times.

\section{Statistical analysis}

Statistic data of each group were determined using GraphPad 6.0. Data were expressed as mean \pm standard deviation (SD). Intergroup difference was evaluated using Student's $t$ test, and multivariate statistical analysis was assessed using Analysis of variance (ANOVA). $P<0.05$ was considered with statistical significance. All experiments were repeated for three times.

\section{Results}

Knockdown of LINC01559 suppresses cell proliferation and promotes cell apoptosis in GC

First, we screened the data from GEPIA2 (http://gepia2. cancer-pku.cn/\#analysis) and found that LINC01559 expression was higher in 408 stomach adenocarcinoma
(STAD) tissues compared with that in 211 normal gastric specimens (Fig. 1A). Simultaneously, data from NCBI (https://www.ncbi.nlm.nih.gov/) indicated that LINC01559 expression was low in normal human stomach tissues (Fig. 1B), which implied that LINC01559 was aberrantly expressed in cancerous situation. Then, the expression level of LINC01559 in four GC cell lines (MKN45, HGC27, SNU-1 and AGS) relative to the human normal gastric epithelial cell line (GES-1) was detected by RT-qPCR analysis. Results manifested that the expression level of LINC01559 in four GC cell lines was significantly upregulated compared with that in GES-1 cells, and its expression in SNU-1 and AGS cells was relatively highest (Fig. 1C). Therefore, these two cancer cell lines were screened out for follow-up experiments. We stably knocked down the expression of LINC01559 in SNU-1 and AGS cells, and confirmed the knockdown efficiency of LINC01559 by sh/LINC01559\#1 and sh/LINC01559\#2 using RT-qPCR (Fig. S1A). Then the data from EdU and colony formation experiments showed that after silencing LINC01559, the proliferation capacity of two cancer cells was significantly reduced (Fig. 1D, E). In contrast, flow cytometry analyzed that in the absence of LINC01559, the apoptosis rate increased by approximately three times (Fig. 1F). The data of following JC-1 assay further verified that silencing of LINC01559 had a promoting effect on the apoptosis of GC cells (Fig. 1G). In short, knockdown of LINC01559 restrains the growth of GC cells.

\section{LINC01559 silencing inhibits GC cell migration and EMT in vitro and hinders tumor growth in vivo}

Subsequently, we further investigated whether LINC01559 affected the behaviors of GC cells regarding metastasis, such as cell migration and EMT. Hence, we conducted transwell experiments and uncovered that the migration ability of GC cells was decreased after the interference of LINC01559 (Fig. 2A). EMT is a biological process in which epithelialderived malignant cells transform into mesenchymal cells, and this process is related to cell migration and invasion ${ }^{22}$. Thus western blot analysis and immunofluorescence experiments were conducted to further verify whether LINC01559 affected EMT process in GC cells. The data from western blot analysis indicated that the expression of E-cadherin (epithelial marker) was increased while the levels of N-cadherin (mesenchymal marker) and EMT-related proteins including Slug, Twist and ZEB1 were decreased after the depletion of LINC01559 in GC cells (Figs. 2B and S1B). Consistently, the results from immunofluorescence experiments further validated that LINC01559 deficiency led to increased E-cadherin expression and reduced $\mathrm{N}$-cadherin expression in both the two GC cells (Fig. 2C). Furthermore, we also carried out in vivo investigations to further test the role of LINC01559 in GC. As expected, silencing of LINC01559 effectively interfered the tumor growth, 


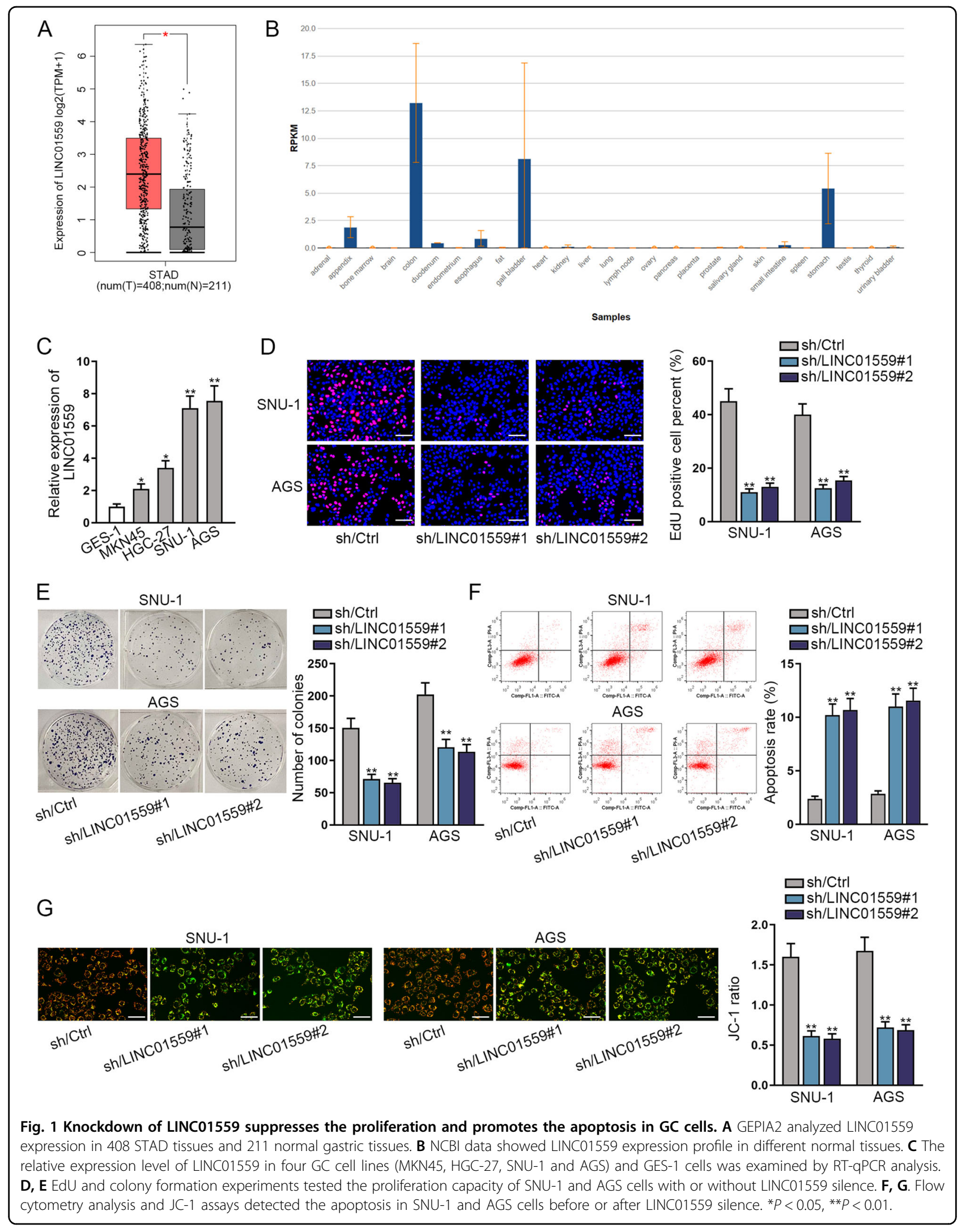




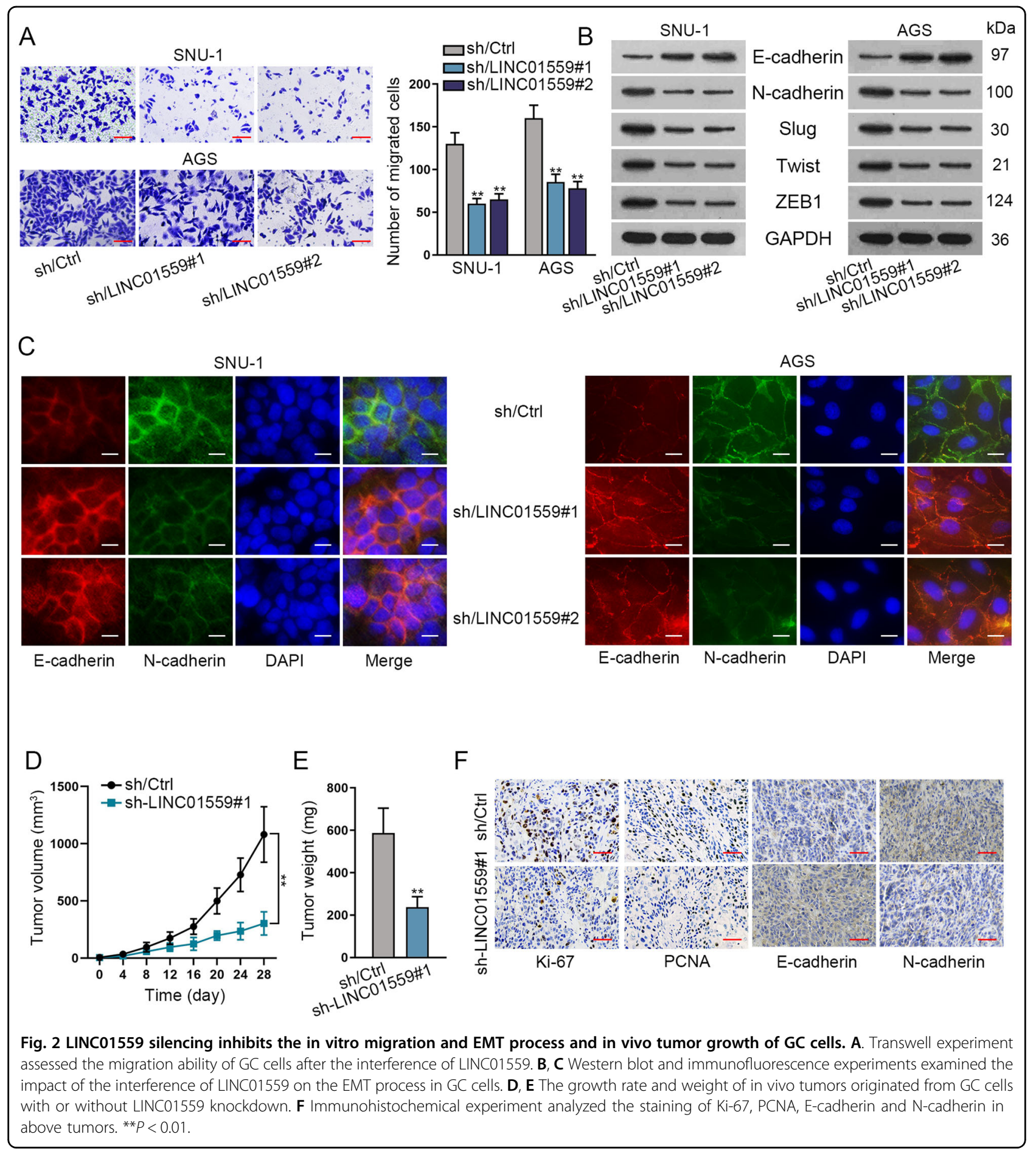

resulting in lighter tumor weight (Fig. 2D, E). In addition, the outcomes of immunohistochemical experiment displayed that the positivity of oncogenic Ki-67, PCNA and Ncadherin was declined while that of tumor-suppressive Ecadherin was enhanced in tumors with the interference of LINC01559 (Fig. 2F). In sum, silencing of LINC01559 inhibits the metastasis and growth of GC cells.

\section{ZEB1 activates LINC01559 expression in GC cells}

Considering the up-regulation of LINC01559 in GC cells and its induction during EMT, we next investigated the transcriptional regulation of LINC01559. Here, we overexpressed several transcription factors in GC cells, and RT-qPCR as well as western blot confirmed the overexpression effectiveness of them in two GC cells 


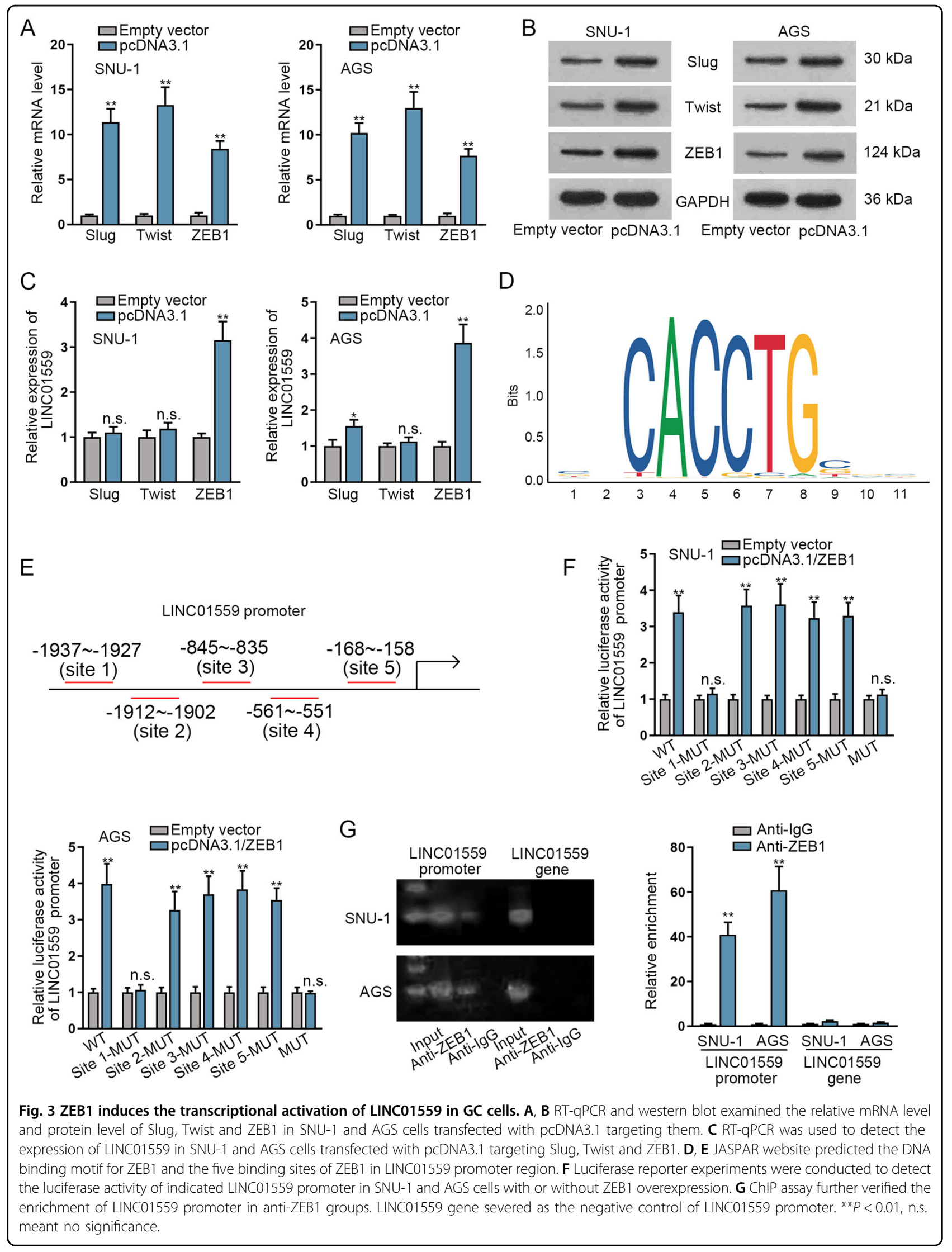




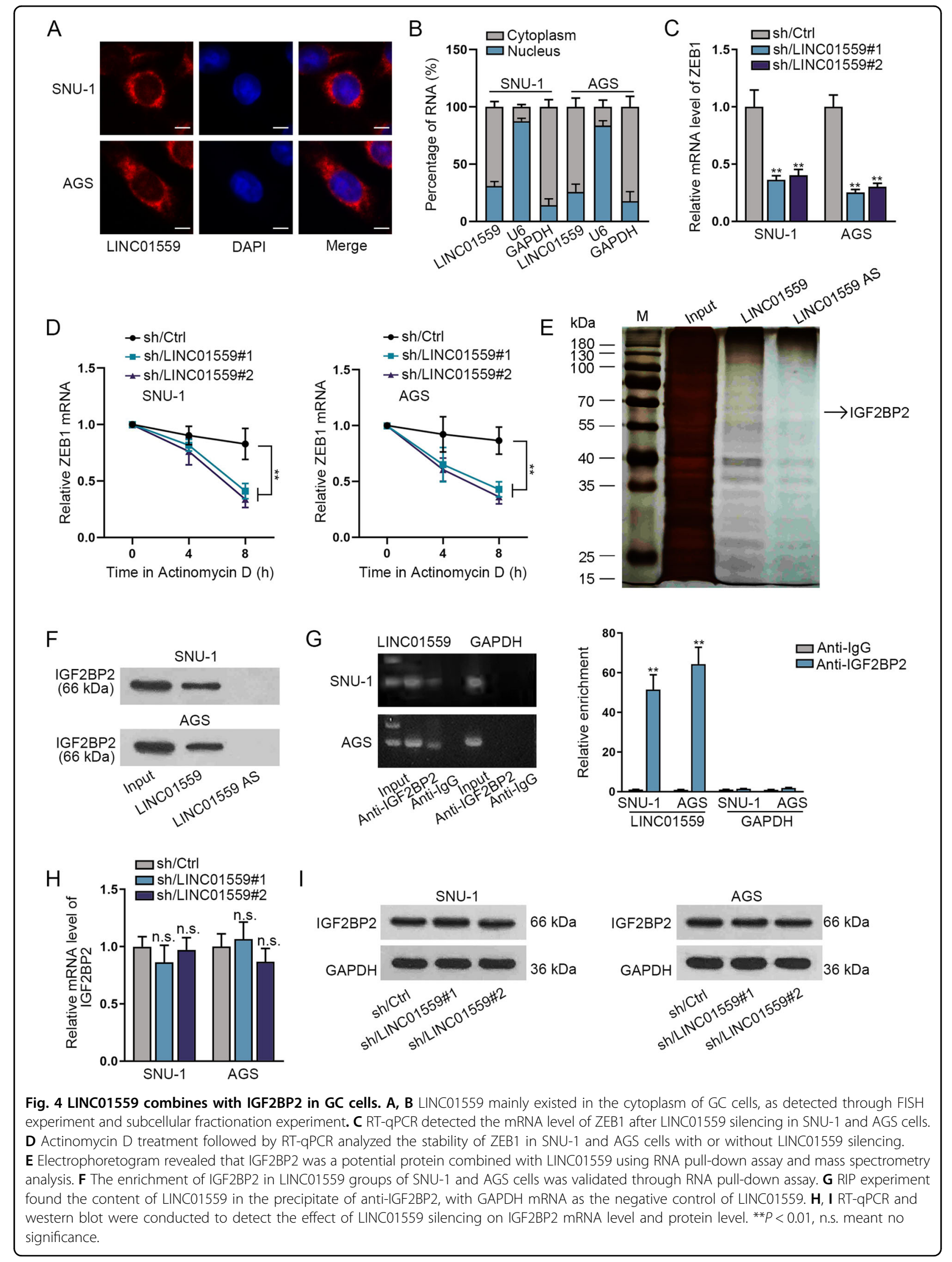


(Figs. 3A, B and S1C, D). Thereafter, RT-qPCR data revealed that the expression level of LINC01559 was significantly up-regulated only by ZEB1 overexpression, while no obvious changes affected by the up-regulation of other transcription factors (Figs. 3C and S1E), suggesting that ZEB1 could activate LINC01559 expression. Then JASPAR website (http://jaspar.genereg.net/) provided the binding motif of ZEB1 and also predicted five binding sites for ZEB1 in LINC01559 promoter (Fig. 3D, E). To further verify the specific interacting location between them, luciferase reporter experiments were conducted. The results displayed that ZEB1 overexpression led to an increase in the luciferase activity of LINC01559 promoter that was wild-type or with site 2/3/4/5 mutations in SNU1 and AGS cells, while no alteration was observed in that of LINC01559 promoter with Site 1 or all five sites mutated (Fig. 3F). This indicated that ZEB1 bound to Site 1 in the LINC01559 promoter. Further, ChIP assay data disclosed that the fragment of LINC01559 promoter region rather than LINC01559 gene region was highly enriched in anti-ZEB1 groups (Fig. 3G), proving the specific combination between LINC01559 promoter and ZEB1 in GC cells. All these results showed that ZEB1 activates the transcription of LINC01559 in GC cells.

\section{LINC01559 combines with IGF2BP2 in GC cells}

After confirming the promoting role of LINC01559 in $\mathrm{GC}$, we further investigated the regulatory mechanism of LINC01559. Before that, we found that LINC01559 mainly existed in the cytoplasm based on the data from FISH experiment and subcellular fractionation experiment (Fig. 4A, B), suggesting that LINC01559 might exert effects in GC cells through post-transcriptional regulation. Interestingly, we detected that the level of ZEB1 was in return decreased by LINC01559 silencing in SNU-1 and AGS cells (Fig. 4C). According to the broken line diagram obtained by PCR and the analysis results of actinomycin D, it could be clearly seen that ZEB1 expression was declined faster after LINC01559 interference (Fig. 4D), reflecting the stability of ZEB1 mRNA was regulated by LINC01559. To investigate how LINC01559 could stabilize ZEB1 mRNA in GC cells, we performed RNA pull-down assay to identify the protein partners of LINC01559. As a result, we focused on a specific band appeared on the electrophoretic gel at about $66 \mathrm{kDa}$ in LINC01559-pulled down products and then found that it was IGF2BP2 protein interacting with LINC01559 after analysis via mass spectrometry (Fig. 4E). We then observed the high enrichment of IGF2BP2 protein in LINC01559 groups through RNA pull-down assay plus an independent immunoblot (Fig. 4F). In addition, according to RIP experimental results, we found that the strong existence of LINC01559 in the precipitate of anti-IGF2BP2 in comparison to the control group (Fig. 4G). Subsequently, RT-qPCR and western blot were conducted to detect whether
LINC01559 could regulate IGF2BP2 expression in GC cells. The experimental results showed that the mRNA level and protein level of IGF2BP2 exhibited no significant difference after the silence of LINC01559 (Fig. 4H, I). Totally, LINC01559 can bind with IGF2BP2 in GC cells.

\section{LINC01559 recruits IGF2BP2 to stabilize ZEB1 mRNA in GC cells}

Then we investigated the interaction between IGF2BP2 and ZEB1 mRNA in GC cells. The data of RNA pull-down assay presented that IGF2BP2 was obviously pulled down by ZEB1 but not by antisense ZEB1 (Fig. 5A). Then, RIP assay showed that ZEB1 was effectively enriched in antiIGF2BP2 groups (Fig. 5B). Notably, the enrichment of ZEB1 in IGF2BP2 groups was dramatically decreased by LINC01559 silencing (Fig. 5C). To determine whether IGF2BP2 could regulate ZEB1, we stably silenced IGF2BP2 expression in GC cells (Fig. 5D). It was found that both the mRNA level and protein level of ZEB1 were obviously declined after the intervene of IGF2BP2 (Fig. 5E). Moreover, the stability of ZEB1 mRNA in GC cells was reduced in response to the interference of IGF2BP2 (Fig. 5F). Therefore, we concluded that LINC01559 recruits IGF2BP2 to stabilize ZEB1 mRNA in GC cells.

\section{LINC01559 promotes GC cell proliferation, migration and EMT process by up-regulating ZEB1 expression}

To further determine whether LINC01559 functioned in GC depending on ZEB1, rescue experiments were carried out. The results of EdU assay and colony formation assay showed that the proliferation of GC cells was significantly weakened after LINC01559 silencing, while such weakening impact was fully counteracted by the co-transfection of pcDNA3.1/ZEB1 (Figs. 6A, B and S2A, B). The data from following flow cytometry analysis and JC-1 experiments showed that the increased apoptotic rate of GC cells induced by LINC01559 silencing could be totally abolished after ZEB1 up-regulation (Figs. 6C, D and S2C, D). Moreover, the results from transwell assays revealed that the lessened number of migrated cells owing to LINC01559 knockdown could be completely rescued by ZEB1 overexpression (Figs. 6E and S2E). Finally, western blot analysis showed that the influence of LINC01559 silencing on the expression of E-cadherin, N-cadherin, Slug, Twist and ZEB1, was thoroughly offset under ZEB1 elevation (Figs. 6F and S2F, G). Together, LINC01559 contributes to GC cell proliferation, migration and EMT by up-regulating ZEB1.

\section{Concept map illustrates the functional role of LINC01559 in GC cells \\ ZEB1-induced LINC01559 facilitates cell proliferation, migration and EMT process in GC through recruiting IGF2BP2 to stabilize ZEB1 expression (Fig. 7).}




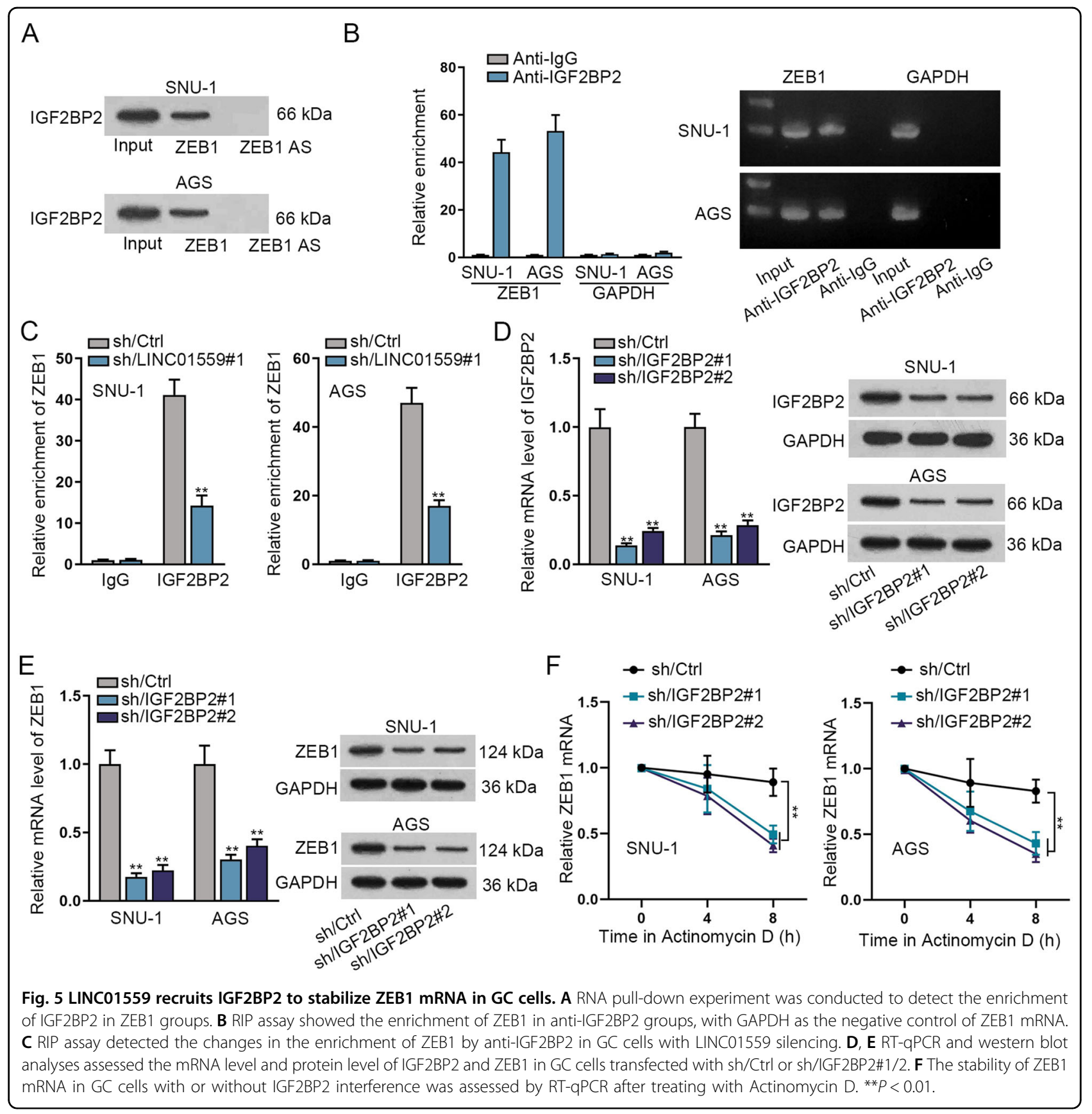

\section{Discussion}

GC is known as being metastatic and invasive ${ }^{23}$. Currently, the therapeutic methods for metastatic GC are very limited, and a lot of efforts have been made to develop new targeted therapies ${ }^{24}$. In this regard, some efforts have been put on the investigation of the relationships between lncRNAs and GC. Fu et al. have proposed that NEAT1 regulates cell proliferation, migration and invasion in $\mathrm{GC}^{25}$. Besides, up-regulation of Sox2ot serves as a poor prognostic marker in GC $^{26}$. Moreover, LINC02163 contributes to GC cell growth and EMT by targeting miR-593-3p to regulate
FOXK1 expression ${ }^{27}$. However, the role of LINC01559 in GC is rarely studied in current literatures. In this study, we found the expression level of LINC01559 was significantly elevated in GC cells. Moreover, we conducted loss-of function assays and found that silencing of LINC01559 obviously inhibited the growth and migration of GC cells, which suggested that LINC01559 could play a tumor promoting role in $\mathrm{GC}$ progression.

It has been reported that ZEB1 is a transcription factor related to the EMT process. Wu et al. have discovered that CASC15 affects cell proliferation, migration and 


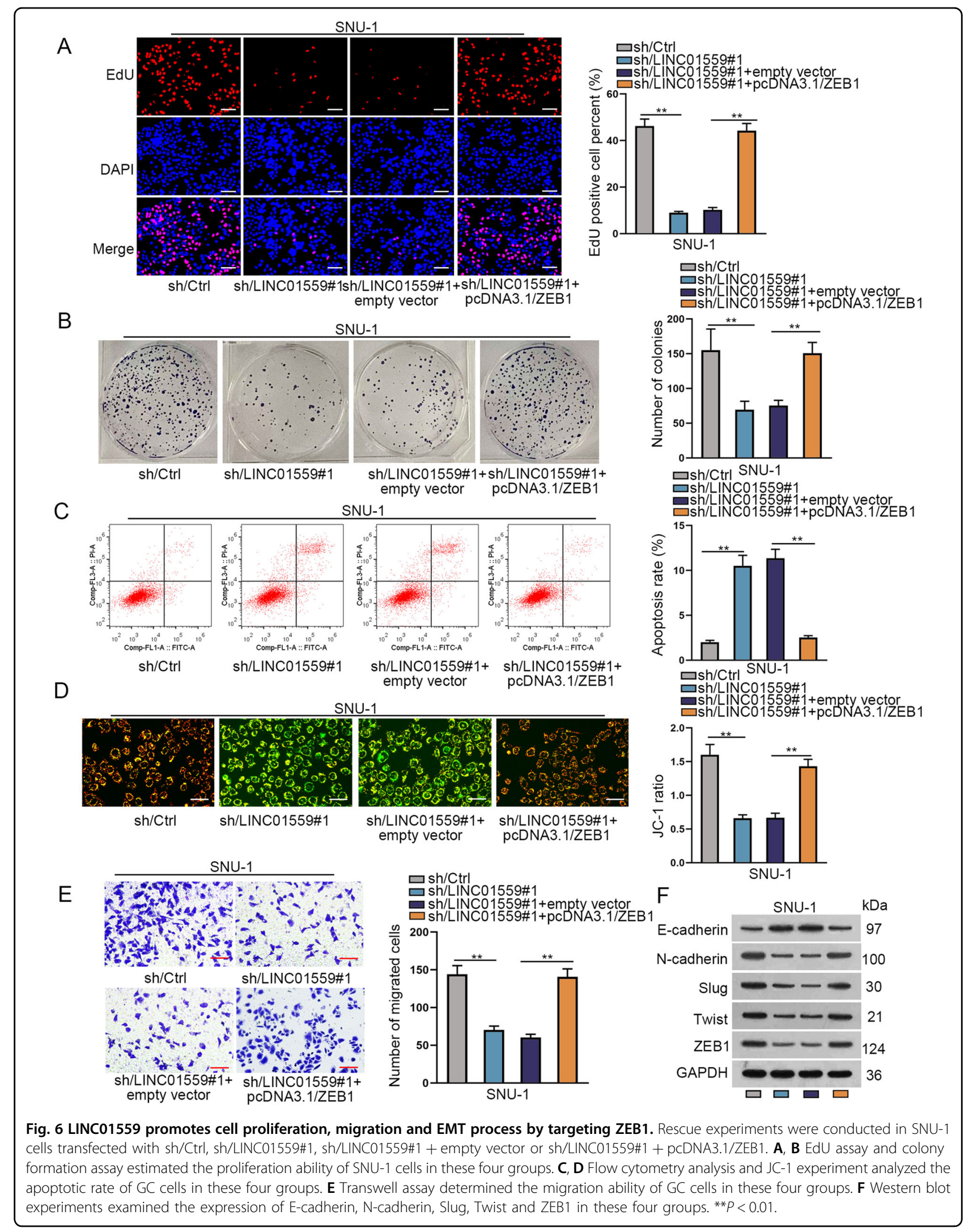




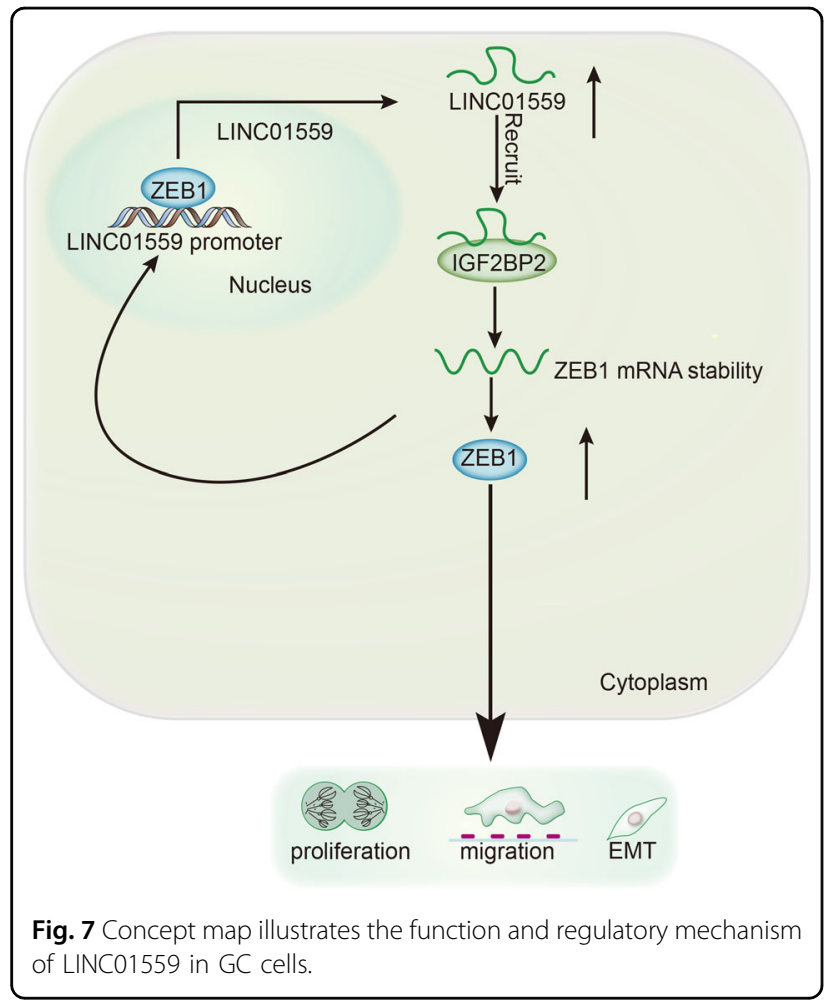

EMT through targeting CDKN1A and ZEB1 in $\mathrm{GC}^{28}$. Besides, silencing of FoxM1 leads to the suppression of EMT in GC cells via regulating ZEB1 ${ }^{29}$. Consistent with these reports, our study confirmed that LINC01559 could regulate the stability of ZEB1 mRNA and impede the EMT process. Notably, our study also found that ZEB1 served as a transcription factor binding to LINC01559 promoter to activate the expression of LINC01559 in GC cells. As reported previously, hypoxiainduced lncRNA-BX111 acts as an oncogene in pancreatic cancer through regulating ZEB1 transcription ${ }^{30}$. ZEB1induced ZEB1-AS1 functions as a tumor-promotor in the progression of non-small cell lung cancer ${ }^{31}$. SOX8 activated by ZEB1 serves as a prognostic factor and development-facilitator in triple-negative breast cancer ${ }^{32}$. All these discoveries are in line with the finding in our study, which provide the basis of the promoting role of LINC01559 in GC cells.

Recent findings have suggested that RBPs are crucial players in post-transcriptional events. Owing to the binding versatility of their RNA-binding domains with structural flexibility, RBPs can modulate the metabolism of a large member of transcripts associated with cancer development ${ }^{17}$. Beside, lncRNAs have been identified to participate in the post-transcriptional control of genes through interacting with $\mathrm{RBPs}^{33}$. Herein, we discovered that LINC01559 mainly distributed in the cytoplasm of GC cells, and therefore we deduced that LINC01559 could have post-transcriptional regulations on genes in GC by interacting with certain RBP.

IGF2BP2 is a type of RBP that plays an important role in tumor development ${ }^{34}$. It has been reported that HOTAIR silencing impedes the invasion and proliferation of human colon cancer cells through regulating IGF2BP2 ${ }^{35}$. MiR$216 \mathrm{~b}$ is implicated in the pathogenesis and progression of hepatocellular carcinoma through regulating IGF2BP2 and $H_{B X}{ }^{36}$. IGF2BP2, as well as KCNQ1 and GCKR, is associated with chemotherapeutic response and survival in patients with metastatic $\mathrm{GC}^{37}$. Similarly, our study screened that IGF2BP2 was certain protein partner of LINC01559. More importantly, we found that IGF2BP2 could combine with ZEB1 and regulate the stability of ZEB1 mRNA. Finally, we carried out a series of rescue experiments and verified that LINC01559 could promote GC cell proliferation, migration and EMT through recruiting IGF2BP2 to stabilize ZEB1 mRNA. As there was still a small proportion of LINC01559 in the nucleus of GC cells, we also wondered its potential role in this part. Reports have recognized that lncRNAs control gene expression via epigenetic or transcriptional regulation in the nucleus ${ }^{38}$. For example, HOTAIR can induce gene silencing through its interplay with $\mathrm{PRC} 2{ }^{39}$. Importantly, a recent study has also verified that LINC01559 can recruit E2H2 to PTEN promoter to activate PI3K/AKT pathway in GC cells ${ }^{40}$. This finding complements the shortcomings of our present work to some extent.

In summary, our study elucidated the oncogenic role of LINC01559 in GC cells and also figured out the LINC01559/IGF2BP2/ZEB1 axis in GC development. Our findings in present study might recommend LINC01559 as a potential therapeutic target for GC.

\section{Acknowledgements \\ Sincere thanks for all supports.}

\section{Author contributions}

H.S. and H.Z. conceived and designed the project. Y.C. and Z.S. performed experiments. W.Q. and C.Q. contributed to data. J.Z. wrote this paper. All authors read and approved final manuscript.

\section{Funding}

This work was supported by Scientific Research project of Shanghai Health Committee (No. 201940433), the project was Involvement of TRIM37 in invasion and metastasis of gastric cancer through ERK signaling pathway, which was received by $H . S$

\section{Conflict of interest}

The authors declare no competing interests.

\section{Ethics statement}

This study was approved by the Ethics Committee of Renji Hospital Affiliated to Medical College of Shanghai Jiaotong University.

\section{Publisher's note}

Springer Nature remains neutral with regard to jurisdictional claims in published maps and institutional affiliations. 
Supplementary information The online version contains supplementary material available at https://doi.org/10.1038/s41419-021-03571-5.

Received: 29 May 2020 Revised: 7 February 2021 Accepted: 1 March 2021 Published online: 06 April 2021

\section{References}

1. Siegel, R. L., Miller, K. D. \& Jemal, A. Cancer statistics, 2018. CA Cancer J. Clin. 68, 7-30 (2018).

2. Torre, L. A. et al. Global cancer statistics, 2012. CA Cancer J. Clin. 65, 87-108 (2015).

3. Chen, W., Zheng, R., Zeng, H., Zhang, S. \& He, J. Annual report on status of cancer in China, 2011. Chin. J. Cancer Res. 27, 2-12 (2015).

4. Cervantes, A., Roda, D., Tarazona, N., Rosello, S. \& Perez-Fidalgo, J. A. Current questions for the treatment of advanced gastric cancer. Cancer Treat. Rev. 39, 60-67 (2013).

5. Shah, M. A. Gastrointestinal cancer: targeted therapies in gastric cancer-the dawn of a new era. Nat. Rev. Clin. Oncol. 11, 10-11 (2014).

6. Niu, Z. S., Niu, X. J. \& Wang, W. H. Long non-coding RNAs in hepatocellular carcinoma: potential roles and clinical implications. World J. Gastroenterol. 23, 5860-5874 (2017)

7. Huo, X. et al. Dysregulated long noncoding RNAs (IncRNAs) in hepatocellular carcinoma: implications for tumorigenesis, disease progression, and liver cancer stem cells. Mol. Cancer 16, 165 (2017).

8. Song, X., Liu, Z. \& Yu, Z. LnCRNA NEF is downregulated in triple negative breast cancer and correlated with poor prognosis. Acta Biochim. et. Biophys. Sin. 51, 386-392 (2019).

9. Yang, J., Du, Y. M. \& Li, B. LncRNA BX357664 inhibits the proliferation and invasion of non-small cell lung cancer cells. Eur. Rev. Med. Pharmacol. Sci. 23 660-669 (2019)

10. Li, C., Liu, T., Zhang, Y., Li, Q. \& Jin, L. K. LncRNA-ZDHHC8P1 promotes the progression and metastasis of colorectal cancer by targeting miR-34a. Eur. Rev. Med. Pharmacol. Sci. 23, 1476-1486 (2019).

11. Yang, $X$. et al. The emergence of long non-coding RNAs in the tumorigenesis of hepatocellular carcinoma. Cancer Lett. 360, 119-124 (2015).

12. Wei, G. H. \& Wang, X. IncRNA MEG3 inhibit proliferation and metastasis of gastric cancer via p53 signaling pathway. Eur. Rev. Med. Pharmacol. Sci. 21, 3850-3856 (2017).

13. Sun, M. et al. LnCRNA HOXA11-AS promotes proliferation and invasion of gastric cancer by scaffolding the chromatin modification factors PRC2, LSD1, and DNMT1. Cancer Res. 76, 6299-6310 (2016).

14. Hu, B., Wang, X. \& Li, L. Long noncoding RNA LINC00337 promote gastric cancer proliferation through repressing p21 mediated by EZH2. Am. J. Transl. Res. 11, 3238-3245 (2019).

15. Orellana-Serradell, O., Herrera, D., Castellon, E. A. \& Contreras, H. R. The transcription factor ZEB1 promotes an aggressive phenotype in prostate cancer cell lines. Asian J. Androl. 20, 294-299 (2018).

16. Maturi, V., Enroth, S., Heldin, C. H. \& Moustakas, A. Genome-wide binding of transcription factor ZEB1 in triple-negative breast cancer cells. J. Cell. Physiol. 233, 7113-7127 (2018).

17. Pereira, B., Billaud, M. \& Almeida, R. RNA-binding proteins in cancer: old players and new actors. Trends Cancer 3, 506-528 (2017).

18. Bao, G., Huang, J., Pan, W., Li, X. \& Zhou, T. Long noncoding RNA CERS6-AS1 functions as a malignancy promoter in breast cancer by binding to IGF2BP3 to enhance the stability of CERS6 mRNA. Cancer Med. 9, 278-289 (2020).

19. Wang, C. et al. A cancer-testis non-coding RNA LIN28B-AS1 activates driver gene LIN28B by interacting with IGF2BP1 in lung adenocarcinoma. Oncogene 38, 1611-1624 (2019).
20. Pastushenko, I. et al. Identification of the tumour transition states occurring during EMT. Nature 556, 463-468 (2018).

21. Barriga, E. H., Franze, K., Charras, G. \& Mayor, R. Tissue stiffening coordinates morphogenesis by triggering collective cell migration in vivo. Nature 554, 523-527 (2018).

22. Hay, E. D. An overview of epithelio-mesenchymal transformation. Acta Anatomica 154, 8-20 (1995).

23. Kadam, P. D. \& Chuan, H. H. Erratum to: Rectocutaneous fistula with transmigration of the suture: a rare delayed complication of vault fixation with the sacrospinous ligament. Int. Urogynecol. J. 27, 505 (2016).

24. Zhou, J. et al. Linc00152 promotes proliferation in gastric cancer through the EGFR-dependent pathway. J. Exp. Clin. Cancer Res. CR 34, 135 (2015).

25. Fu, J. W., Kong, Y. \& Sun, X. Long noncoding RNA NEAT1 is an unfavorable prognostic factor and regulates migration and invasion in gastric cancer. J. Cancer Res. Clin. Oncol. 142, 1571-1579 (2016).

26. Zhang, Y., Yang, R., Lian, J. \& Xu, H. LncRNA Sox2ot overexpression serves as a poor prognostic biomarker in gastric cancer. Am. J. Transl. Res. 8, 5035-5043 (2016).

27. Dong, L. et al. LINC02163 regulates growth and epithelial-to-mesenchymal transition phenotype via miR-593-3p/FOXK1 axis in gastric cancer cells. Artif. Cells Nanomed. Biotechnol. 46, 607-615 (2018).

28. $\mathrm{Wu}, \mathrm{Q}$. et al. Long non-coding RNA CASC15 regulates gastric cancer cell proliferation, migration and epithelial mesenchymal transition by targeting CDKN1A and ZEB1. Mol. Oncol. 12, 799-813 (2018).

29. Miao, L. et al. Down-regulation of FoxM1 leads to the inhibition of the epithelial-mesenchymal transition in gastric cancer cells. Cancer Genet. 207, 75-82 (2014).

30. Deng, S. J. et al. Hypoxia-induced LncRNA-BX111 promotes metastasis and progression of pancreatic cancer through regulating ZEB1 transcription. Oncogene 37, 5811-5828 (2018).

31. Qu, R., Chen, X. \& Zhang, C. LncRNA ZEB1-AS1/miR-409-3p/ZEB1 feedback loop is involved in the progression of non-small cell lung cancer. Biochem. Biophys. Res. Commun. 507, 450-456 (2018).

32. Tang, $\mathrm{H}$. et al. SOX8 acts as a prognostic factor and mediator to regulate the progression of triple-negative breast cancer. Carcinogenesis 40, 1278-1287 (2019).

33. Lan, Y. et al. Long noncoding RNA OCC-1 suppresses cell growth through destabilizing HuR protein in colorectal cancer. Nucleic Acids Res. 46, 5809-5821 (2018).

34. Dahlem, C., Barghash, A., Puchas, P., Haybaeck, J. \& Kessler, S. M. The insulin-like growth factor 2 mRNA binding protein IMP2/IGF2BP2 is overexpressed and correlates with poor survival in pancreatic cancer. Int. J. Mol. Sci. 20, https://doi. org/10.3390/ijms20133204 (2019).

35. Wu, X. L., et al. Long noncoding RNA HOTAIR silencing inhibits invasion and proliferation of human colon cancer LoVo cells via regulating IGF2BP2. J. Cell. Biochem., https://doi.org/10.1002/jcb.27079 (2018).

36. Liu, F. Y. et al. MiR-216b is involved in pathogenesis and progression of hepatocellular carcinoma through HBx-miR-216b-IGF2BP2 signaling pathway. Cell Death Dis. 6, e1670 (2015).

37. Liu, X. et al. Effects of IGF2BP2, KCNQ1 and GCKR polymorphisms on clinical outcome in metastatic gastric cancer treated with EOF regimen. Pharmacogenomics 16, 959-970 (2015).

38. Yao, R. W., Wang, Y. \& Chen, L. L. Cellular functions of long noncoding RNAs Nat. Cell Biol. 21, 542-551 (2019).

39. Portoso, M. et al. PRC2 is dispensable for HOTAIR-mediated transcriptional repression. EMBO J. 36, 981-994 (2017).

40. Wang, L. et al. Exosome-transferred LINC01559 promotes the progression of gastric cancer via PI3KJAKT signaling pathway. Cell Death Dis. 11, 723, (2020). 